\title{
6 An Adaptive Virtual Gallery
}

In this chapter we present a design scenario that applies the generative design grammar described in Chapter 5 within a Generative Design Agent (GDA). The design scenario consists of eight different stages. These different stages present various situations where changes may occur in a virtual gallery during its use; for example, changes of activities, changes of exhibition requirements, changes of visitors, and so on. The design scenario demonstrates that the artist's GDA reasons about these changes and dynamically generates and modifies the design of the virtual gallery to accommodate the changes. Although the design scenario is constructed with a specific kind of virtual gallery in mind, it demonstrates the effective use of GDAs and generative design grammars for rule-based place design in $3 \mathrm{D}$ virtual worlds.

\subsection{Design Scenario}

The characters in the design scenario include the artist, the guest and various visitors. The artist is represented as a Generative Design Agent (GDA) in the virtual gallery. Other characters are represented as general avatars, which only have the visual representation as the $3 \mathrm{D}$ animated characters but have no agency - the ability to reason, design and act in the virtual gallery. The GDA on the other hand can reason, design and act on behalf of the artist in the virtual gallery. The design component of the GDA is supported by the application of the example generative design grammar. The eight stages of the design scenario present situations where various changes occur in the virtual gallery; for example, changes of the artist's and other visitors' activities, changes of the artist's exhibitions, increase and decrease of visitor numbers, and so on. The GDA senses the changes and hypothesizes design goals in order to accommodate these changes. To meet these design goals, the GDA applies the example grammar to generate different designs of the virtual gallery as needed.

\subsubsection{Purpose of the Design Scenario}

The purpose of the scenario is to demonstrate the application of the GDA and the example generative design grammar for rule-based design of a virtual gallery that adapts to its use. The design scenario shows how a GDA reasons, designs and acts in the virtual gallery for some typical situations where changes occur in the gallery during its use. The scenario does not intend to address all possible situations and changes, although they can be considered and addressed in future development.

(c))BY-NC-ND 2014 Ning Gu and Mary Lou Maher This work is licensed under the Creative Commons Attribution-NonCommercial-NoDerivs 3.0 License. 
An ideal agent-based virtual world should be represented by a society of GDAs where the designs of the world are dynamically generated by the multi-agent society, so that the designs meet the common goals of the virtual community and reflect their common interests. To do so, the communication among GDAs needs to be addressed. Although the GDA model is applicable to multi-agent virtual worlds, since the reasoning mechanism of the GDA model enables each GDA to reason about the virtual world as well as other GDAs in the virtual world, agent communication is however beyond the scope of this book. To control the complexity of the scenario, as mentioned earlier, only the artist is represented with a GDA in the virtual gallery.

- When the artist is present in the virtual gallery, the virtual gallery will be dynamically designed, implemented and manipulated by the GDA, on behalf of the artist. To a certain extent, the visitors' needs and interests in the virtual gallery are also reflected in the generated designs, as the design goals of the GDA are hypothesized based on its interpretations of the virtual gallery and different users' activities in the gallery.

- When the artist is not present, the virtual gallery will be replaced by a static design. Static and adaptive virtual galleries are further discussed below.

\subsubsection{Generative Design Grammar Application}

The application of the example generative design grammar is directed by a set of special state labels in order to generate designs that meet the GDA's current design goals. Each design rule of the example grammar is associated with one of these state labels. A design rule is applied only when the following conditions are met.

- The LHO of the rule is recognized in the virtual gallery, and

- The design context represented by the state label is related to the GDA's current design goals.

However, in cases where there is more than one design rule that meets the above conditions a control mechanism is needed to resolve the conflict. In general, there are three main methods for controlling the generative design grammar application. They are random selection, human designer or user intervention and agent learning mechanism.

- The random selection method allows the system to randomly select one design rule from the set of rules that meet the conditions.

- The human designer or user intervention method allows the system to turn to human designers or users for instructions once such a conflict occurs.

- The agent learning mechanism provides a more autonomous but more complex approach to allow the system to resolve such a conflict based on the GDA's previous design experiences and user feedback. 
The example grammar applies the human designer or user intervention method. When the artist's GDA finds more than one design rule that can be applied, the GDA informs the artist and waits for further instructions. In the design scenario, such a case occurs twice, one at Stage 2 and the other at Stage 5.

\subsubsection{Static and Adaptive Virtual Gallery}

In the design scenario, the virtual gallery has static and adaptive designs. The scenario starts with the login of the artist to the virtual world, where the artist's virtual gallery is located. The artist is represented by a GDA in the virtual world. This marks the beginning of the virtual gallery being dynamically designed, implemented and manipulated as needed by the GDA, adapting to its use. As the artist is the only character of the scenario given the agency in the virtual gallery, adaptive virtual gallery therefore is not possible without their presence. Prior to the start of the scenario, a static design of the virtual gallery is used in the virtual world. The design is the result of the artist's previous visit. As shown in Figure 6.1, this static design comprises one gallery area for displaying one of the exhibitions. The gallery area connects to a reception area from floor 1 of the reception area. Without the GDA, the virtual gallery is static because the reasoning and designing activities are supported through the GDA.

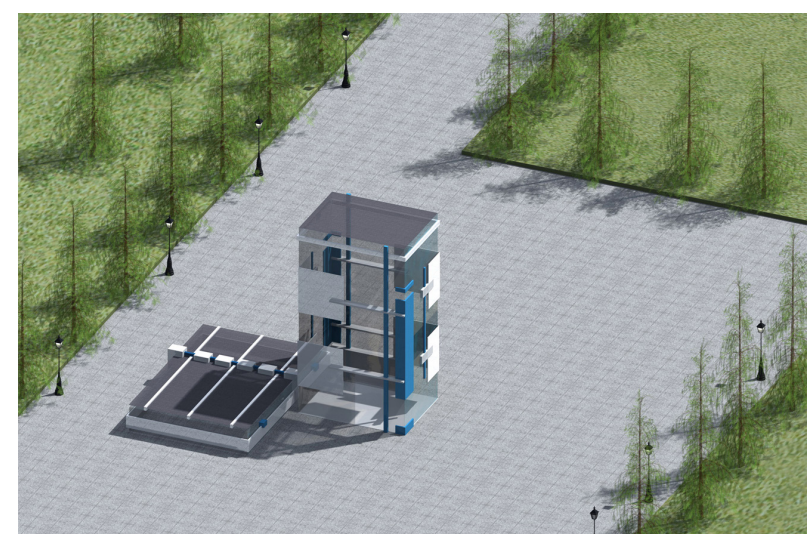

Figure 6.1: The visualization of the static virtual gallery design used prior to the design scenario.

At the end of the design scenario, the artist disconnects from the virtual world. The GDA applies the termination rule to terminate the grammar application before terminating its own agent program. As a result, a static design of the virtual gallery is generated once again. The dynamic designing process will re-start when the artist returns to the virtual gallery. 


\subsection{Stage 1: The Artist Enters the Virtual Gallery}

At Stage 1, the artist connects to the virtual world, where the artist's virtual gallery is located. The artist is represented by a GDA in the virtual world. At the moment, he intends to display two new exhibitions: exhibitions 1 and 2 in the virtual gallery. In the initial static virtual gallery, the GDA senses a number of visitors, each of whom are represented by an avatar. On behalf of the artist, the GDA welcomes the visitors and notifies them that the virtual gallery will be temporarily under construction for arranging new exhibitions. The visitors are reminded that in a very short moment they will be invited and transported to the new exhibitions.

The GDA, meanwhile, demolishes the initial static virtual gallery and at the same location places the initial design of the example grammar: the layout of a reception area. With the initial design being recognized, the virtual gallery's dynamic designing process starts.

\subsubsection{Layout Rule Application}

The application of the design rules in a generative design grammar follows the sequence of layout rules, object design rules, navigation rules and interaction rules.

To prepare for the application of layout rules at Stage 1, The GDA performs the following reasoning:

- In the process of interpretation, the GDA interprets (1) the presence of the artist, and that (2) the artist currently has no studio space in the virtual gallery. The GDA also interprets that (3) the artist intends to display two exhibitions: exhibitions 1 and 2, and (4) currently the virtual gallery has no gallery space available.

- In the process of hypothesizing, based on interpretation (1) and (2), the GDA hypothesizes a design goal $\mathrm{O}_{\exp }^{\mathrm{F}}=\mathrm{S}$ (the personal studio area for the artist is needed in the virtual gallery). Based on interpretation (3) and (4), the GDA hypothesizes the next two design goals $\mathrm{O}_{\exp }{ }^{\mathrm{F}}=\mathrm{g} 1$ (the initial standard gallery 1 area is needed in the virtual gallery) and $\mathrm{O}_{\exp }{ }^{\mathrm{F}}=\mathrm{g} 2$ (the initial standard gallery 2 area is needed in the virtual gallery).

With these design goals being hypothesized, the GDA starts the rule matching process in order to apply the example grammar:

- For matching the LHOs of the design rules: the initial design $\left.{ }^{\circ}\right]$ is recognized.

- For matching the state labels of the design rules: because $\mathrm{O}_{\exp }{ }^{\mathrm{F}}=\mathrm{S}, \mathrm{O}_{\exp }^{\mathrm{F}}=\mathrm{g} 1$ and $\mathrm{O}_{\exp }{ }^{\mathrm{F}}=\mathrm{g} 2$ are hypothesized, therefore $\mathrm{sL}=\mathrm{S}, \mathrm{sL}=\mathrm{g} 1$ and $\mathrm{sL}=\mathrm{g} 2$ are matched.

Based on the above criteria, the GDA searches the design rules of the example grammar for eligible rules to apply, starting from layout rules. As each layout rule is associated 
with state label $\mathrm{sL}=1$ that indicates the stage of the grammar application, the matched state labels become $\mathrm{sL}=1 \mathrm{~S}, \mathrm{sL}=1 \mathrm{~g} 1$ and $\mathrm{sL}=1 \mathrm{~g} 2$. The matched layout rules are:

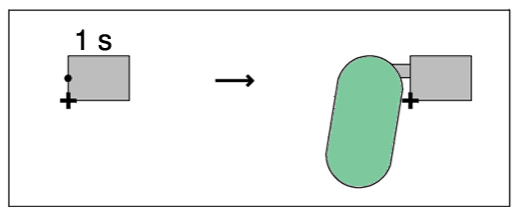

Match 1: additive layout rule 1.

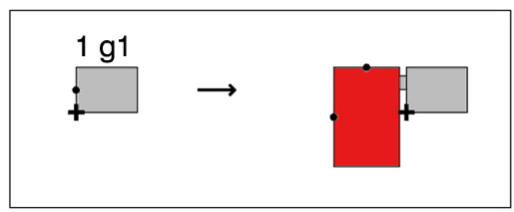

Match 2: additive layout rule 2.

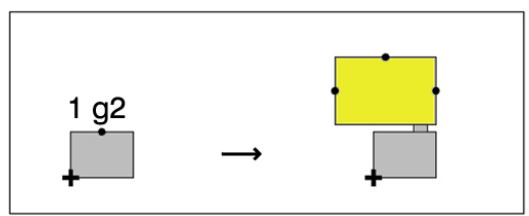

Match 3: additive layout rule 3.

The application of the above layout rules generates a layout

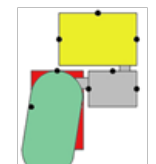

for the virtual gallery, which comprises a reception area, a personal studio area for the artist, a standard gallery 1 area for displaying exhibition 1 , and a standard gallery 2 area for displaying exhibition 2.

\subsubsection{Object Design Rule Application}

Object design rules are the second set of design rules to be applied.

Now that the layout of the virtual gallery is generated, additional reasoning is required by the GDA to continue with the application of object design rules. In order to generate visual boundaries and visual cues for each area, the GDA needs to be provided with the artist's design preferences for the interior of the virtual gallery and the specifications of the exhibitions.

- In the process of interpretation, based on the instructions given by the artist, the GDA interprets that (1) the artist prefers the use of a cold-color scheme for the interior of the virtual gallery, and (2) exhibitions 1 and 2 each contains various 
digital images in various sizes which can be arranged for displaying in the virtual gallery using configuration 1 .

- In the process of hypothesizing, based on interpretation (1), the GDA hypothesizes a design goal $\mathrm{O}_{\exp }{ }^{\mathrm{F}}=\mathrm{cC}$ (to apply a cold-color scheme for the interior of the virtual gallery). Based on interpretation (2), the GDA hypothesizes the next design goal $\mathrm{O}_{\exp }{ }^{\mathrm{F}}=\mathrm{gIM} 1$ (to arrange the two gallery areas for displaying digital images using configuration 1$)$.

With these design goals being hypothesized, the GDA starts the matching process:

- For matching the LHOs of the design rules: $\square, U, \square$ and $\square$, the layouts of the four areas are recognized.

- For matching the state labels of the design rules: because $\mathrm{O}_{\exp }{ }^{\mathrm{F}}=\mathrm{CC}$ and $\mathrm{O}_{\exp }{ }^{\mathrm{F}}=\mathrm{gIM} 1$ are hypothesized, therefore $\mathrm{sL}=\mathrm{cC}$ and $\mathrm{sL}=\mathrm{gIM} 1$ are matched.

Based on the above criteria, the GDA continues the search in object design rules for eligible rules to apply. As each object design rule is associated with state label $\mathrm{sL}=2$ that indicates the stage of the grammar application, the matched state labels become $\mathrm{sL}=2 \mathrm{cC}$ and $\mathrm{sL}=2 \mathrm{gIM}$. The matched object design rules are:
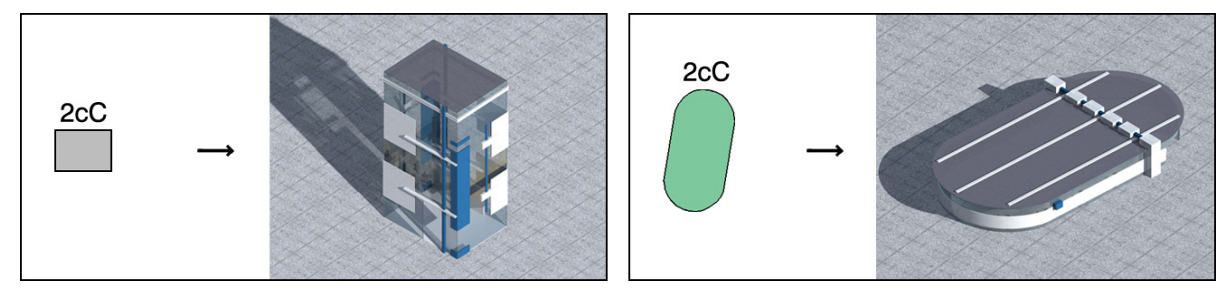

Matches 1 and 2: additive object design rules 1 (left) and 2 (right).
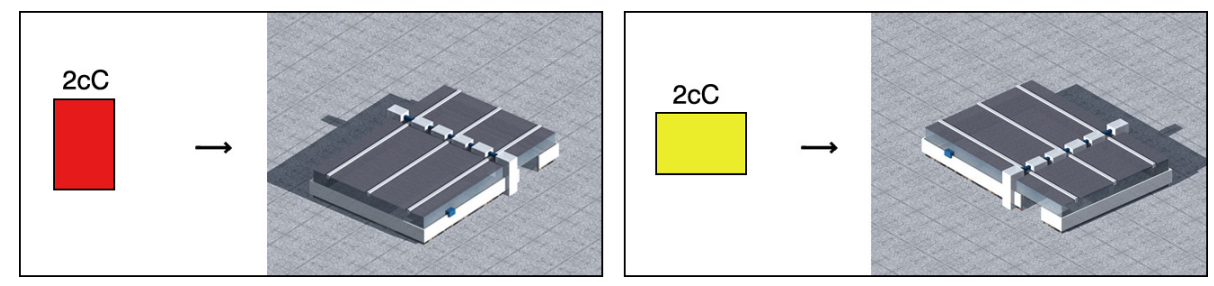

Matches 3 and 4: additive object design rules 3 (left) and 4 (right).

The application of these four object design rules provides the generated 2D layout of the virtual gallery with purposeful 3D objects to define visual boundaries and provide visual cues for each area. Subsequently, for the two gallery areas, the following two object design rules are also matched to further arrange the gallery areas for displaying the two exhibitions: 


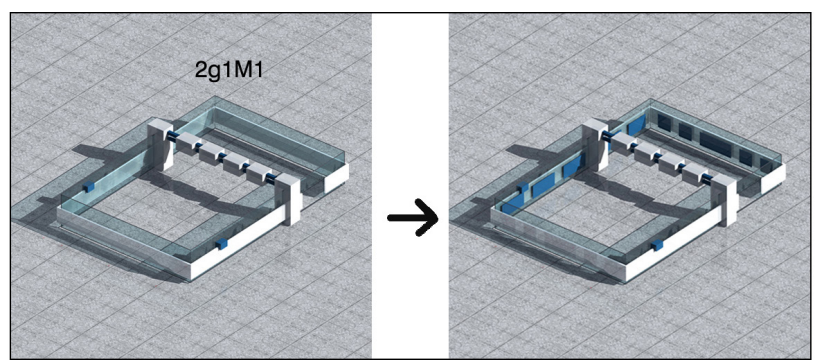

Match 5: additive object design rule 8.

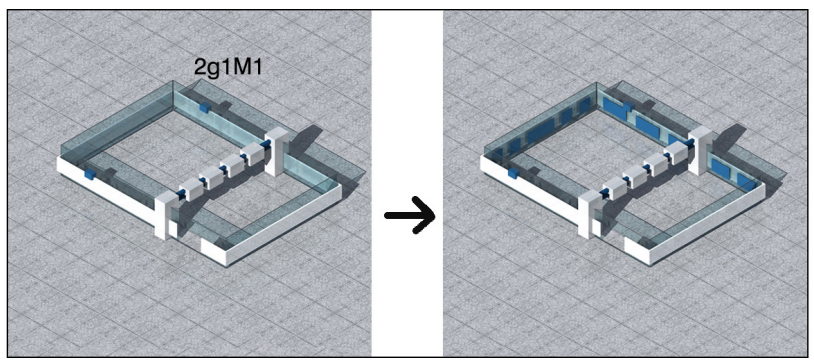

Match 6: additive object design rule 9.

The result of the object design rule application is shown in Figure 6.2. The virtual gallery has four areas: the reception area, the artist's personal studio area, a standard gallery 1 area and a standard gallery 2 area. The reception area has three floors and connects to gallery 1 from floor 1, gallery 2 from floor 2, and the studio area from floor 3.

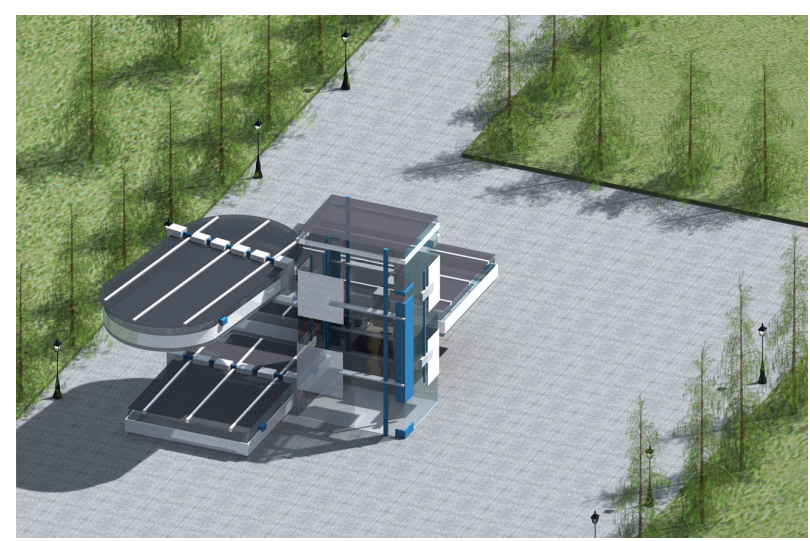

Figure 6.2: The visualization of the virtual gallery design for Stage 1. 


\subsubsection{Navigation Rule Application}

Next, the GDA applies navigation rules to provide way finding aids and hyperlinks ${ }^{12}$ to the generated design of the virtual gallery. Navigation rules are the third set of design rules to be applied after layout rules and object design rules. In the matching process:

- For matching the LHOs of the design rules: the four areas of the virtual gallery generated at Stage 1 are recognized. They are shown in Figure 6.3 from left to right: the reception area, the artist's personal area, the standard gallery 1 area and the standard gallery 2 area.

- For matching the state labels of the design rules: the design goal $\mathrm{O}_{\exp }{ }^{\mathrm{F}}=\mathrm{gIM} 1$ is related to not only the application of object design rules, but also the application of navigation rules. Therefore, because $\mathrm{O}_{\exp }{ }^{\mathrm{F}}=\mathrm{gIM} 1$ is hypothesized, $\mathrm{SL}=\mathrm{gIM} 1$ is hence matched.
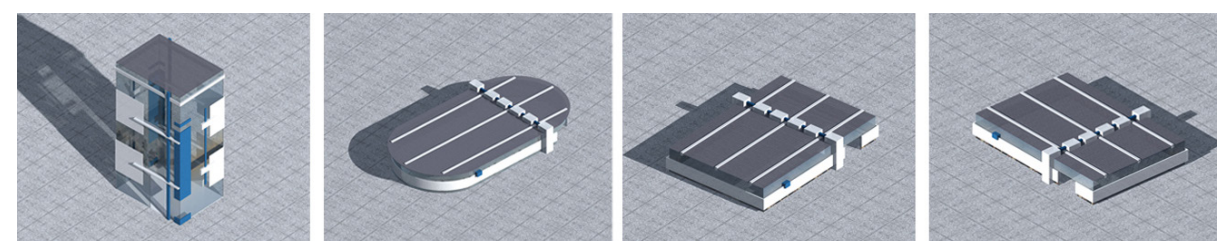

Figure 6.3: The visualizations of the four areas of the virtual gallery generated at Stage 1.

Based on the above criteria, the GDA searches navigation rules of the example grammar, and the matched navigation rules are:

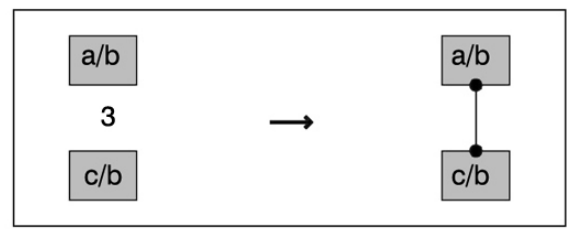

Match 1: additive navigation rule 3.

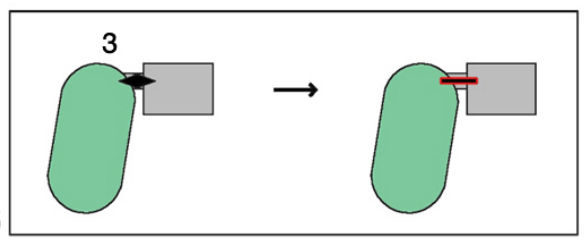

Match 2: additive navigation rule 18.

12 The guidelines for using way finding aids and hyperlinks in this virtual gallery are presented in Chapter 5. 


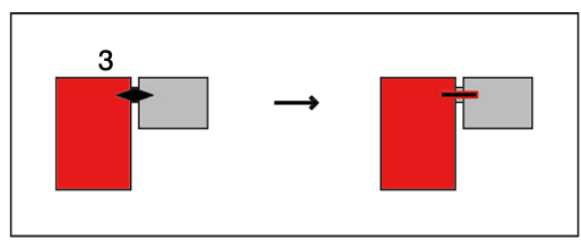

Match 3: additive navigation rule 19.

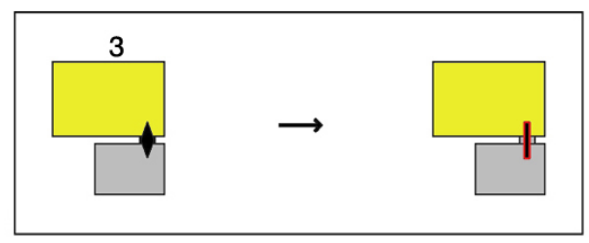

Match 4: additive navigation rule 20.

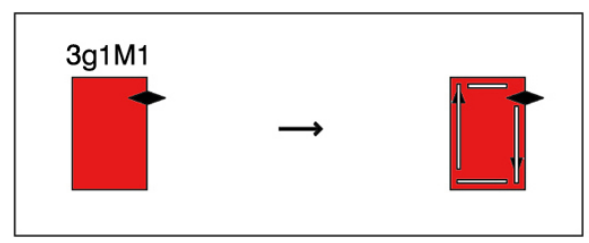

Match 5: additive navigation rule 4.

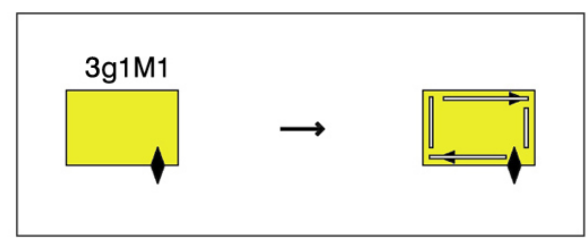

Match 6: additive navigation rule 5.

Additive navigation rule 3 is applied multiple times to connect the three floors of the reception area together with each other using hyperlinks. Additive navigation rules 4 and 5 are applied to lay paths in the two gallery areas for guiding visitors through the exhibitions. Finally, additive navigation rules 18, 19 and 20 are applied to lay paths to connect the reception area with the artist's personal studio area, and the two gallery areas, for directing visitors.

\subsubsection{Interaction Rule Application}

To complete the design for Stage 1, the GDA applies interaction rules to ascribe scripted behaviors to selected objects in the virtual gallery. Therefore, visitors can interact with the environment by triggering these behaviors and to participate in various intended 
activities. Interaction rules are non-visual/spatial rules, they are about recognizing selected objects in the virtual gallery and ascribing appropriate behaviors to these objects. Therefore, in the matching process, the main concern is to match the LHOs of the design rules. The only state label used in all interaction rules is $\mathrm{sL}=4$ indicating they are the fourth, the final set of design rules to be applied.

The GDA recognizes various digital picture frame objects in the two gallery areas for displaying digital images of the exhibitions, various digital document objects in the reception area and the artist's personal studio area for storing and redirecting digital information, and various hyperlinks on different floors of the reception area. Based on these criteria, the GDA searches interaction rules of the example grammar, and the matched interaction rules are:

\section{Match 1: additive interaction rule 1:}

\section{$\mathrm{SL}=4$}

IF: The 3D model of a digital picture frame object is recognized within a gallery area.

AND

The digital picture frame object is currently not configured.

THEN: Render the appropriate digital image onto the surface of the 3D model from the artist's exhibition. AND

Enable the digital image to be enlarged and accessed from the web browser.

\section{Match 2: additive interaction rule 3:}

\section{$\mathrm{SL}=4$}

IF: The 3D model of a digital document object is recognized within a reception area, the artist's personal studio area, or the multi-function area.

AND

The digital document object is currently not configured.

THEN: Attach the relevant digital information to the object.

AND

Enable the detail of the information to be accessed from the web browser.

\section{Match 3: additive interaction rule 6:}

\section{$\mathrm{SL}=4$}

IF: The 3D models of a pair of hyperlinks are recognized connecting two different floors of a reception area.

AND

The hyperlinks are currently not configured.

THEN: Detect the coordinates of the hyperlinks.

AND

Detect any obstacle between these two locations.

IF: No obstacle exists.

THEN: Activate the hyperlinks using the detected coordinates.

IF: Any obstacle exists.

THEN: Change the hyperlinks.

AND

Activate the hyperlinks using the detected coordinates. 
Additive interaction rule 1 is applied multiple times to display digital images that form the two exhibitions using various digital picture frame objects. Additive interaction rule 3 is applied multiple times to provide information about the virtual gallery and the exhibitions in the reception area, and to store the artist's digital tools and data in his personal studio area and also to redirect to other media, using various digital document objects. Additive interaction rule 6 is applied multiple times to activate the hyperlinks so that the visitors can navigate through the three floors of the reception area to access different parts of the virtual gallery.

The final design of the virtual gallery for Stage 1 is now generated with four areas: the reception area that provides information regarding the virtual gallery and the exhibitions, the personal studio area equipped with various digital tools and data for the artist, and two gallery areas that are configured for displaying the artist's two exhibitions. In the processes of action, the GDA plans actions for implementing this generated design of the virtual gallery, and activates the planned actions via its effectors in the virtual world. Once the design for Stage 1 is implemented, the GDA notifies the artist and the visitors before transporting the artist to the personal studio area and the visitors to the gallery areas.

For the remaining stages of the scenario, the execution of each stage follows similar procedures shown above at Stage 1. In the following sections, these procedures are described in a simpler version.

\subsection{Stage 2: Exhibition 1 Receives More Visitors}

At Stage 2, more visitors connect to the virtual world and visit the virtual gallery. At one point, the number of visitors in the standard gallery 1 reaches the maximum capacity of a standard gallery area ${ }^{13}$. The artist's GDA senses this change and applies the example grammar to add an additional gallery area for displaying exhibition 1. Any future visitors who wish to visit exhibition 1 will be automatically transported to this newly generated gallery area, until the number of visitors in the original gallery area drops below the maximum number.

\subsubsection{Execution of the Design Scenario}

The execution of Stage 2 follows similar procedures shown at Stage 1. In the process of interpretation, the GDA interprets the changing needs of the artist and the visitors and changes in the virtual gallery; for example, the initial change at Stage 2 is the increase of visitors in the standard gallery 1 area. Based on its current interpretations,

13 For this example grammar, a gallery area has two different sizes and each gallery area is designed for hosting a certain number of avatars for the comfort of the visitors' viewing and the ease of their avatars' movements in the area. 
in the process of hypothesizing, the GDA hypothesizes design goals. For example, one of the design goals hypothesized by the GDA at Stage 2 is $\mathrm{O}_{\text {exp }} \mathrm{F}=\mathrm{g} 1+$ (an additional standard gallery 1 area is needed in the virtual gallery). With new design goals being hypothesized, new state labels can be matched for the search of eligible design rules in the example grammar for application.

Firstly, in the application of layout rules the newly matched state label is $\mathrm{sL}=1 \mathrm{~g} 1+$. The following two layout rules both satisfy the criteria:

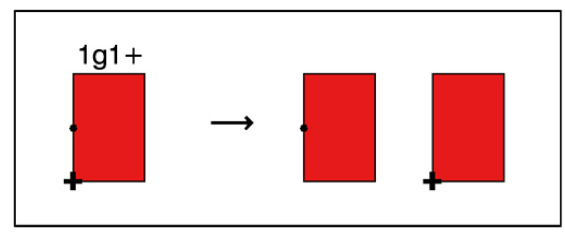

Additive layout rule 4.

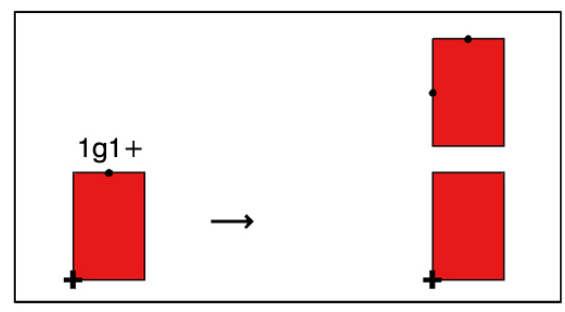

Additive layout rule 6.

Both layout rules add an additional gallery area in the virtual gallery. Additive layout rule 4 expands the layout of the virtual gallery along the $\mathrm{X}$ axis (local to the design), and additive layout rule 6 expands the layout along the $\mathrm{Y}$ axis (local to the design). The GDA turns to the artist for further instructions. In the design scenario, the artist prefers to expand the layout of the virtual gallery along the Y axis, therefore the GDA applies additive layout rule 6 for design generation.

With the application of other relevant layout rules, a layout

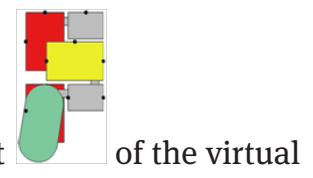
gallery is generated for Stage 2 of the design scenario.

Secondly, in the application of object design rules the newly matched state labels are $\mathrm{sL}=2 \mathrm{cC}$ and $\mathrm{sL}=2 \mathrm{gIM} 1$. The matched object design rules are:

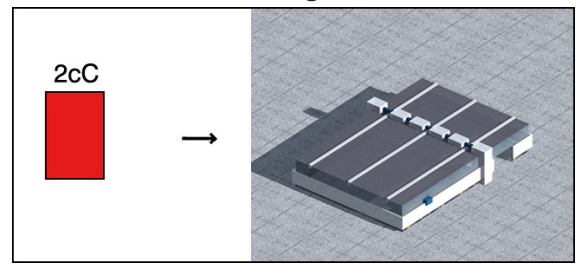

Additive object design rule 3. 


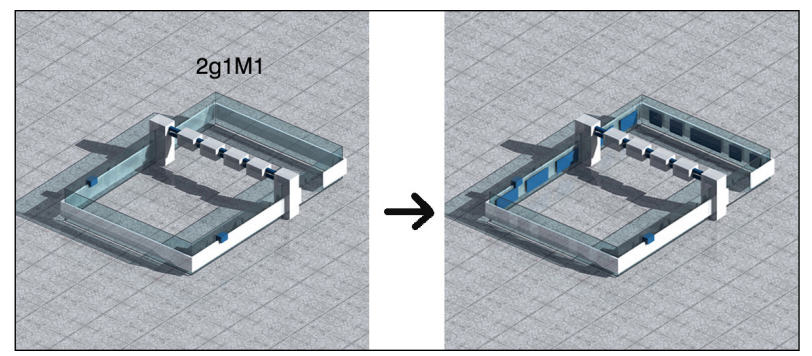

Additive object design rule 8.

Additive object design rule 3 is applied to define visual boundaries and provide visual cues for the newly generated gallery area, and this gallery area is further arranged for displaying exhibition 1 by applying additive object design rule 8 . The result of the object design rule application is shown in Figure 6.4.

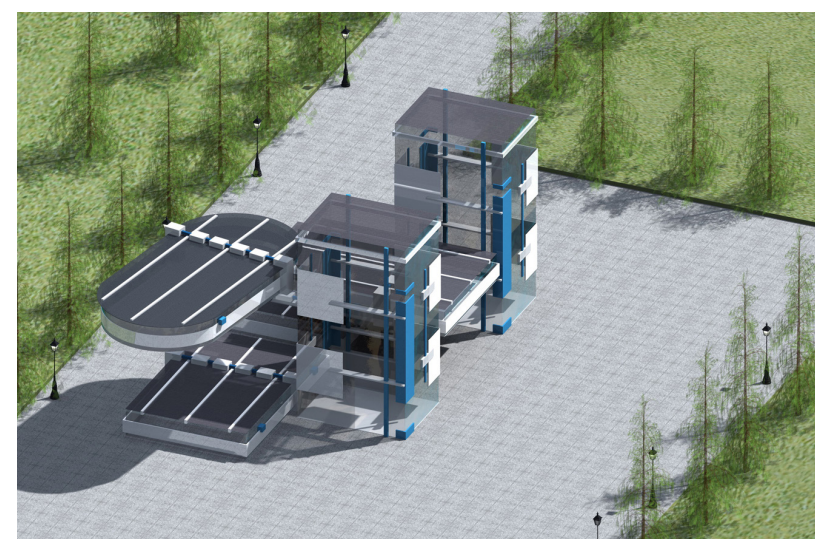

Figure 6.4: The visualization of the virtual gallery design for Stage 2.

To complete the design, the GDA searches and applies appropriate navigation rules to provide way-finding aids and hyperlinks to connect with the newly generated areas. Finally, the GDA searches and applies appropriate interaction rules to ascribe scripted behaviors to selected objects in the newly generated areas when it is necessary.

Compared to the design generated for Stage 1, the current design has an additional gallery area (a standard gallery 1 area) for displaying exhibition 1. An additional reception area is also generated to connect the newly generated gallery area with the rest of the virtual gallery. 


\subsubsection{An Alternative Design of the Virtual Gallery for Stage 2}

As mentioned earlier, the application of layout rules for Stage 2 can have two different ways to expand the virtual gallery. An alternative design could have been generated if the artist had preferred to expand the virtual gallery along the $\mathrm{X}$ axis.

If this had been the case, additive layout rule 4 (see page 107) would be applied,

and the layout generated for Stage 2 would be

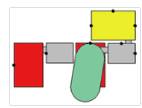
would change the design of the virtual gallery for Stage 2, and subsequently change all the designs generated for the rest of the design scenario.

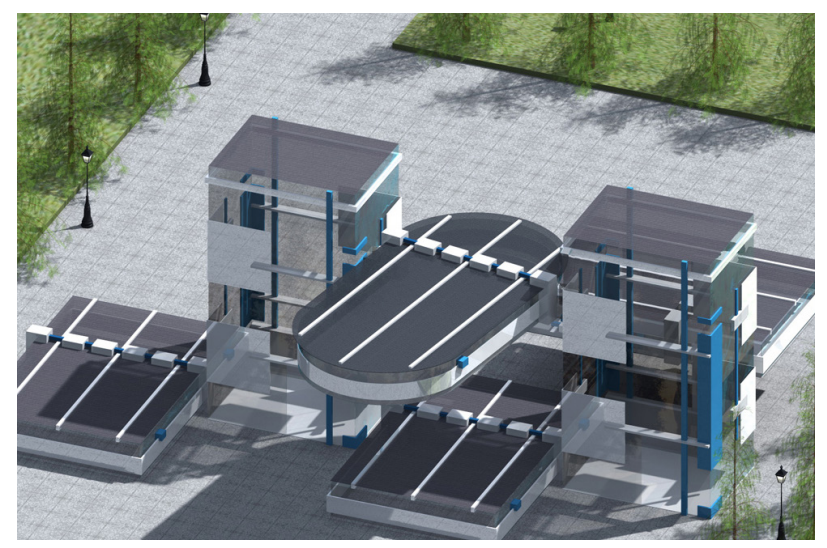

Figure 6.5: The visualization of the alternative design at Stage 2.

\subsection{Stage 3: An Invited Guest Enters the Virtual Gallery}

At Stage 3, the artist is browsing some digital information in his personal studio area. He instructs his GDA to organize a meeting venue for meeting his guest $\mathrm{B}$, who will be arriving in the virtual gallery soon. The GDA applies the example grammar to arrange a meeting area inside the artist's personal studio area. When user B connects to the virtual world, the GDA welcomes B on behalf of the artist and transports B's avatar directly to the newly-generated meeting area.

For the execution of Stage 3, in the process of interpretation, the GDA interprets the changing needs of the artist and the visitors and changes in the virtual gallery; for example, the changing need of the artist at Stage 3 reflects the request for a meeting venue. Based on its current interpretations, in the process of hypothesizing, the GDA hypothesizes a design goal $\mathrm{O}_{\exp }^{\mathrm{F}}=\mathrm{mS}$ (to configure a meeting area in the artist's 
personal studio area). With the new design goal being hypothesized, new state labels can be matched for the search of eligible design rules in the example grammar for application.

To configure a meeting area in the artist's personal studio area does not require the application of layout rules since the personal studio area has been generated at Stage 1 . In the application of object design rules, the newly matched state label is $\mathrm{sL}=2$ $\mathrm{mS}$. The matched object design rule is:

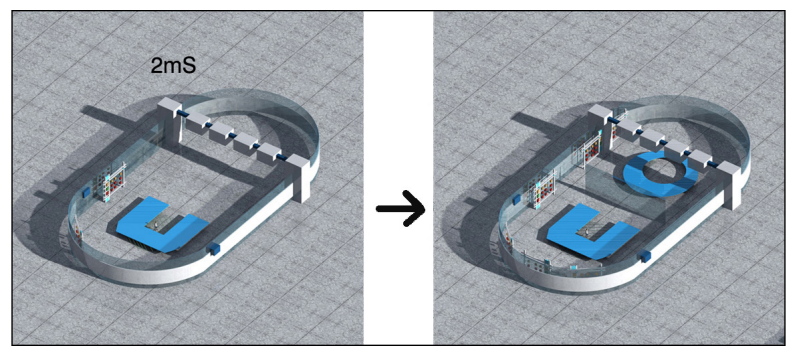

Additive object design rule 22.

As the meeting area is arranged inside the artist's personal studio area it is not necessary to provide way finding aids and hyperlinks separately for this area. Therefore, navigation rules are also not applied at Stage 3. To complete the design, the GDA searches and applies appropriate interaction rules to ascribe scripted behaviors to activate various meeting facilities in the newly arranged meeting area.

The current design of the virtual gallery is the same as the design generated for Stage 2, except that a meeting area is now arranged inside the artist's personal studio area.

\subsection{Stage 4: The Artist Prepares for an Opening Address}

At Stage 4, the artist decides to give an opening address to highlight the newly opened exhibitions after the meeting with the guest. The artist instructs the GDA to organize a venue for the public function. The GDA applies the example grammar to generate the multi-function area in the virtual gallery and arranges the area as a conference venue. After the artist finishes the preparation for the talk, the GDA sends an invitation to the visitors on behalf of the artist and transports those who accept the invitation to the multi-function area.

For the execution of Stage 4, in the process of interpretation, the GDA interprets the changing needs of the artist and the visitors and changes in the virtual gallery. For 
example, the changing need of the artist at Stage 4 reflects the request for a venue to host a public talk. Based on its current interpretations, in the process of hypothesizing, the GDA hypothesizes design goals; for example, one of the design goals hypothesized by the GDA at Stage 4 is $\mathrm{O}_{\exp }{ }^{\mathrm{F}}=\mathrm{mC}$ (the multi-function area is needed as a conference venue). With new design goals being hypothesized, new state labels can be matched for the search of eligible design rules in the example grammar for application.

Firstly, in the application of layout rules, the newly matched state label is $\mathrm{SL}=1$ $\mathrm{mC}$. The matched layout rule is:

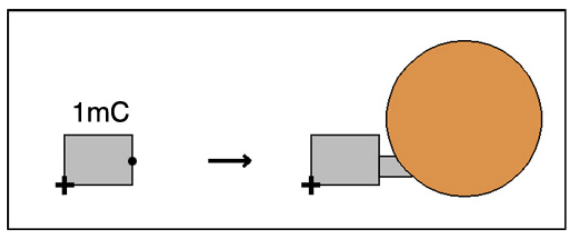

Additive layout rule 14.

By applying this layout rule, a layout

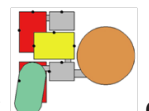
for Stage 4. The multi-function area is added.

Secondly, in the application of object design rules the newly matched state labels are $\mathrm{sL}=2 \mathrm{cC}$ and $\mathrm{sL}=2 \mathrm{mMc}$. The matched object design rules are:

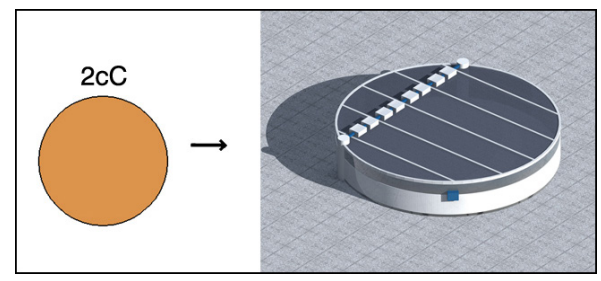

Additive object design rule 7.

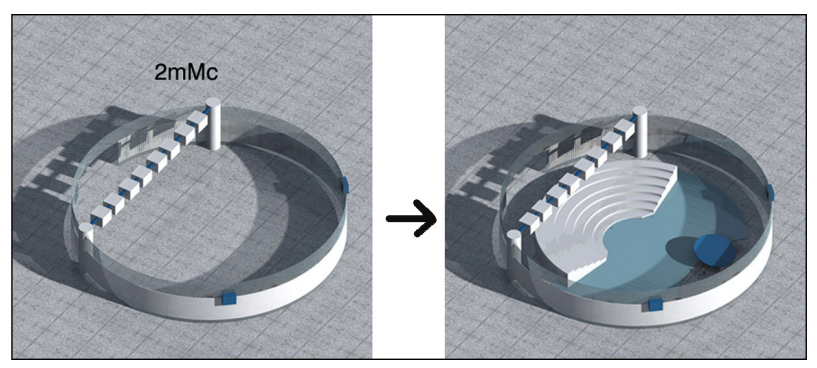

Additive object design rule 23.

Additive object design rule 7 is applied to define visual boundaries and provide visual cues for the newly generated multi-function area, and this area is further arranged as 
a conference venue by applying additive object design rule 23 . The result of the object design rule application is shown in Figure 6.6.

To complete the design, the GDA searches and applies appropriate navigation rules to provide way finding aids and hyperlinks to connect with the multi-function area, and, finally, the GDA searches and applies appropriate interaction rules to ascribe scripted behaviors to activate various conference facilities in the multi-function area.

Compared to the design generated for Stage 3, the current design of the virtual gallery has a venue for public functions, that is, the multi-function area is arranged as a conference venue for the artist's opening address.

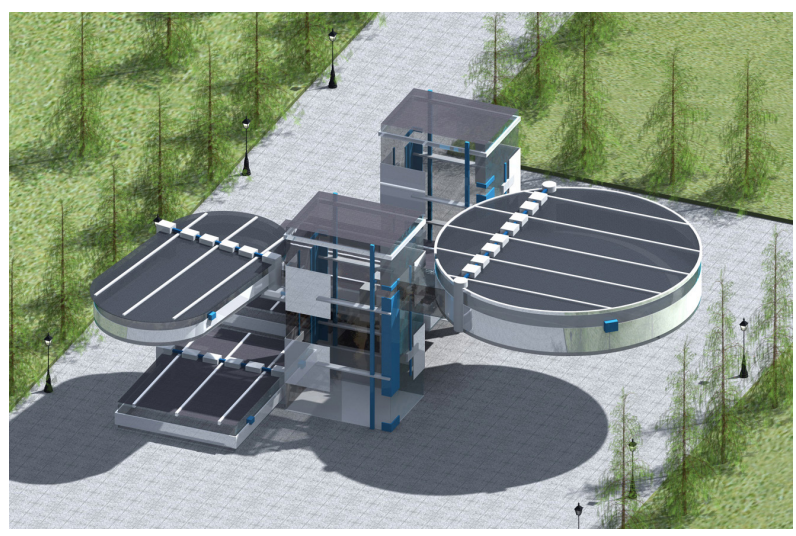

Figure 6.6: The visualization of the virtual gallery design for Stage 4.

\subsection{Stage 5: Exhibition 2 Receives More Visitors}

After the talk some visitors remain in the multi-function area for discussion with the artist. The rest return to the exhibitions. At one point, the number of visitors in the standard gallery 2 area also reaches the maximum capacity of a standard gallery area. The artist's GDA senses this change and applies the example grammar to add an additional gallery area for displaying exhibition 2 . Any future visitors who wish to visit exhibition 2 will be automatically transported to this newly generated gallery area until the number of visitors in the original gallery area drops below the maximum number.

\subsubsection{Execution of the Design Scenario}

For the execution of Stage 5, in the process of interpretation, the GDA interprets the changing needs of the artist and the visitors and changes in the virtual gallery; for 
example, the initial change at Stage 5 is the increase of visitors in the standard gallery 2 area. Based on its current interpretations, in the process of hypothesizing, the GDA hypothesizes design goals; for example, one of the design goals hypothesized by the GDA at Stage 5 is $\mathrm{O}_{\exp }{ }^{\mathrm{F}}=\mathrm{g} 2+$ (an additional standard gallery 2 area is needed in the virtual gallery). With new design goals being hypothesized, new state labels can be matched for the search of eligible design rules for application.

Stage 5 is very similar to Stage 2, where a design goal $\mathrm{O}_{\text {exp }}^{\mathrm{F}}=\mathrm{g} 1+$ (an additional standard gallery 1 area is needed in the virtual gallery) was hypothesized. Following the same procedures shown at Stage 2, the GDA matches new states labels, searches and applies appropriate layout rules, object design rules, navigation rules and interaction rules to generate an additional gallery area to accommodate more visitors for exhibition 2.

The application of layout rules generates a layout

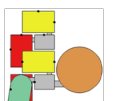
for Stage 5. The design of the virtual gallery for this stage is shown in Figure 6.7 Compared to the design generated for Stage 4, the current design of the virtual gallery has an additional gallery area (a standard gallery 2 area) for displaying exhibition 2.

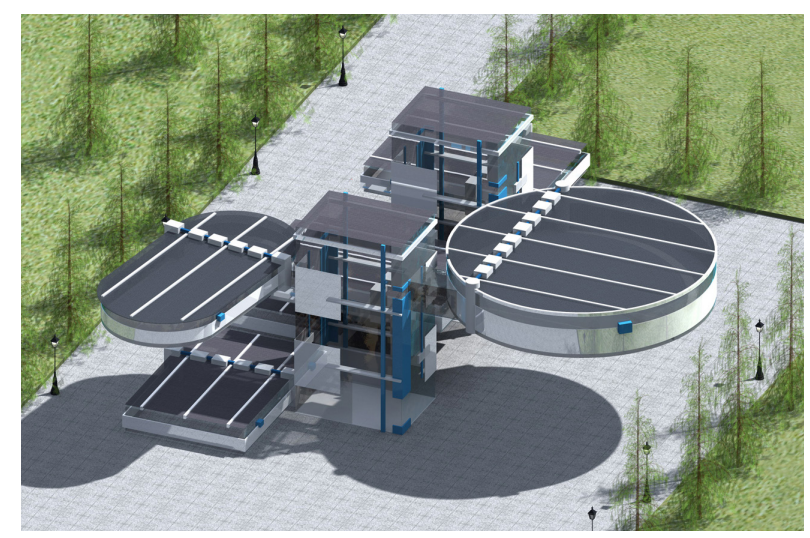

Figure 6.7: The visualization of the virtual gallery design for Stage 5 .

\subsubsection{Alternative Designs of the Virtual Gallery for Stage 5}

Similar to Stage 2, the application of layout rules for Stage 5 can also have two different ways to expand the virtual gallery by applying the following two rules: 


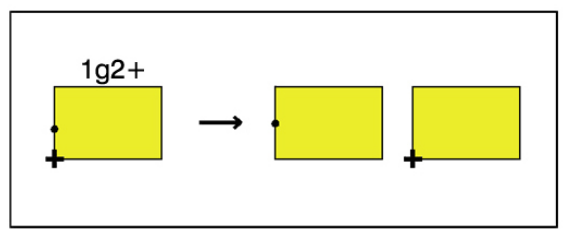

Additive layout rule 5

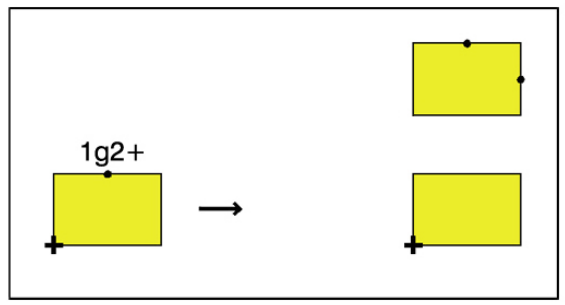

Additive layout rule 7

Additive layout rule 5 expands the layout of the virtual gallery along the $\mathrm{X}$ axis (local to the design), while additive layout rule 7 expands the layout along the $Y$ axis (local to the design). In the scenario, the artist once again prefers to expand the layout of the virtual gallery along the $\mathrm{Y}$ axis, therefore the GDA applies additive layout rule 7 for design generation.

For both Stages 2 and 5, as discussed, there are two different ways to expand the layout of the virtual gallery. Figure 6.8 shows the two different layouts of the virtual gallery that can be generated for Stage 2. Based on these two layouts for Stage 2 the subsequent stages can each have two different layouts generated for the virtual gallery. The two layouts of the virtual gallery that can be generated for Stage 4 are illustrated in Figure 6.9.
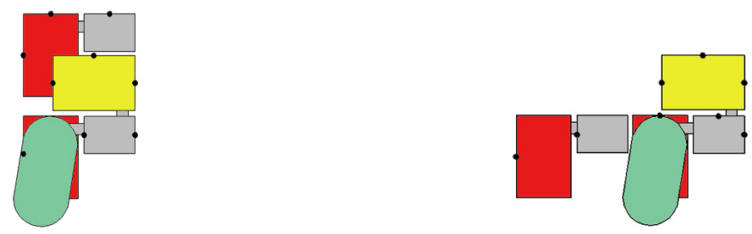

Figure 6.8: The two layouts that can be generated for Stage 2 .
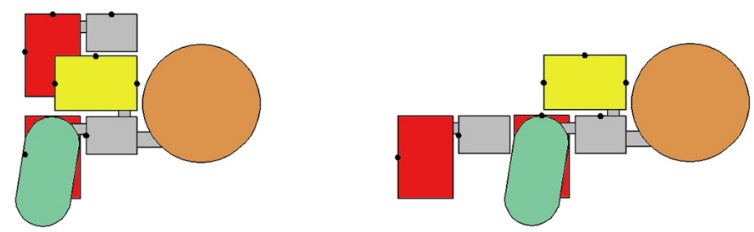

Figure 6.9: The two layouts that can be generated for Stage 4. 
Based on the two layouts shown in Figure 6.9, by alternating the application of additive layout rules 5 and 7 three alternative layouts of the virtual gallery can be generated for Stage 5, besides the one generated for this design scenario. The alternative layouts are illustrated in Figure 6.10.

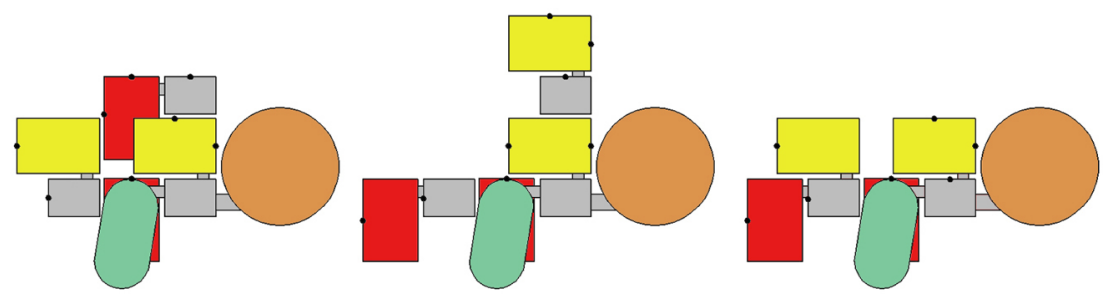

Figure 6.10: The three alternative layouts that can be generated for Stage 5.

\subsection{Stage 6: The Artist Changes Exhibition Requirements}

At Stage 6, the artist returns to his personal studio area and decides to modify exhibition 2 by adding more exhibition items. The artist instructs the GDA to accommodate the changes. On behalf of the artist, the GDA informs the visitors about the changes and applies the example grammar to expand and rearrange both gallery 2 areas in order to display the new exhibition 2 .

For the execution of Stage 6, in the process of interpretation, the GDA interprets the changing needs of the artist and the visitors and changes in the virtual gallery; for example, the changing need of the artist at Stage 6 reflects the changes of exhibition 2. Based on its current interpretations, in the process of hypothesizing, the GDA hypothesizes design goals; for example, one of the design goals hypothesized by the GDA at Stage 6 is $\mathrm{O}_{\text {exp }}^{\mathrm{F}}=\mathrm{gE} 2$ (the two standard gallery 2 areas need to be expanded). With new design goals being hypothesized, new state labels can be matched for the search of eligible design rules in the example grammar for application.

Firstly, in the application of layout rules, the newly matched state label is $\mathrm{SL}=1$ gE2. The matched layout rules are:

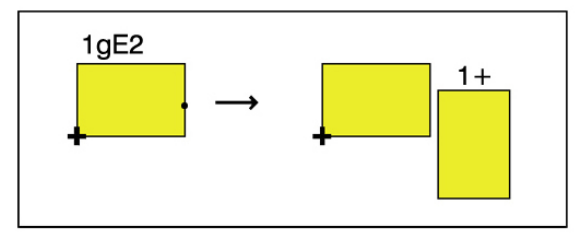

Additive layout rule 12. 


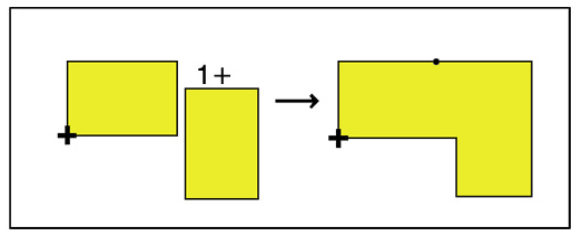

Additive layout rule 13.

By applying the above rules, a layout

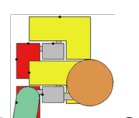
of the virtual gallery is generated for Stage 6. The two standard gallery 2 areas are expanded.

Secondly, in the application of object design rules, the newly matched state labels are $\mathrm{sL}=2 \mathrm{cC}$ and $\mathrm{sL}=2 \mathrm{gIMS}$. The matched object design rules are:

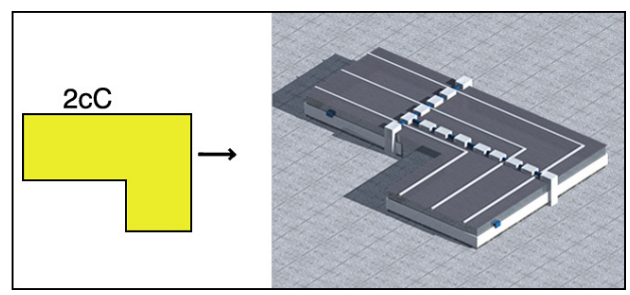

Additive object design rule 6.

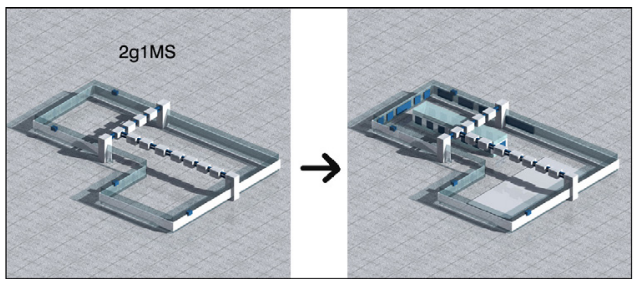

Additive object design rule 21.

Additive object design rule 6 is applied to define visual boundaries and provide visual cues for the newly generated expanded gallery 2 areas ${ }^{14}$, and the areas are further arranged for displaying the new exhibition 2 by applying additive object design rule 21. The result of the object design rule application is shown in Figure 6.11.

To complete the design, the GDA searches and applies appropriate navigation rules to provide way finding aids and hyperlinks to connect with the expanded gallery 2 areas, and, finally, the GDA searches and applies appropriate interaction rules to

14 At Stage 6, before the application of additive object design rules, various subtractive object design rules are applied to remove excessive objects from the previous design. 
ascribe scripted behaviors to selected objects in these newly generated areas, when it is necessary.

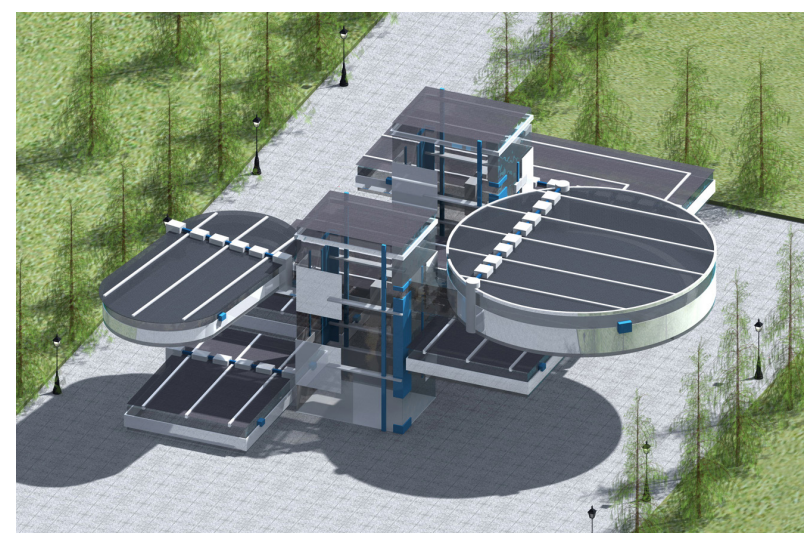

Figure 6.11: The visualization of the virtual gallery design for Stage 6.

Compared to the design generated for Stage 5, the current design of the virtual gallery has expanded the two gallery 2 areas for displaying the new exhibition 2 which has more exhibition items.

\subsection{Stage 7: Some Visitors Leave the Virtual Gallery}

At Stage 7, more and more visitors disconnect from the virtual world. At one point, one of the gallery 1 areas has no visitor. The artist' GDA senses this change and applies its generative design grammar to remove this gallery area. Similar situations soon occur in one of the gallery 2 areas and in the multi-function area.

For the execution of Stage 7, in the process of interpretation, the GDA interprets the changing needs of the artist and the visitors and changes in the virtual gallery; for example, the main change at Stage 7 is the decrease of visitors in the virtual gallery. Based on its current interpretations, in the process of hypothesizing, the GDA hypothesizes design goals. For example, one of the design goals hypothesized by the GDA at Stage 7 is $\mathrm{O}_{\exp }{ }^{\mathrm{F}}=\mathrm{g} 1-$ (a standard gallery 1 area is redundant). With new design goals being hypothesized, new state labels can be matched for the search of eligible design rules in the example grammar for application.

Firstly, in the application of layout rules the newly matched state labels are $\mathrm{sL}=1$ g1-, sL=1 g2- and sL=1 m-. The matched layout rules are: 


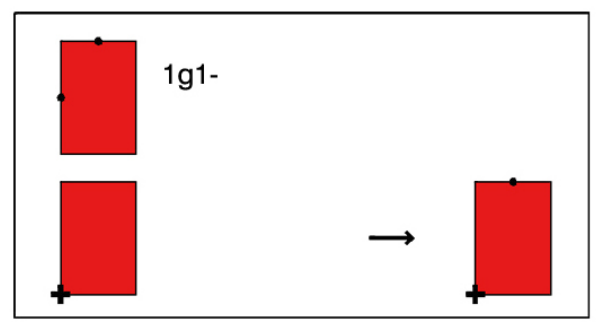

Subtractive layout rule 3.

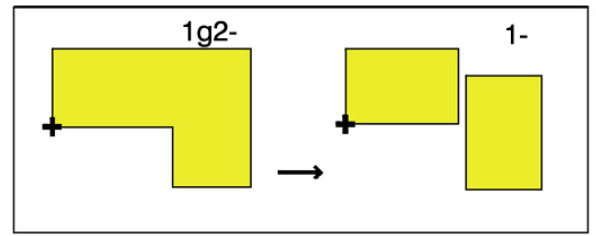

Subtractive layout rule 6.

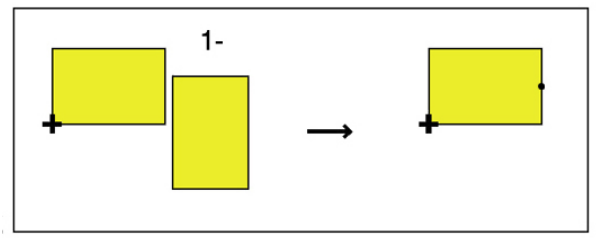

Subtractive layout rule 12 .

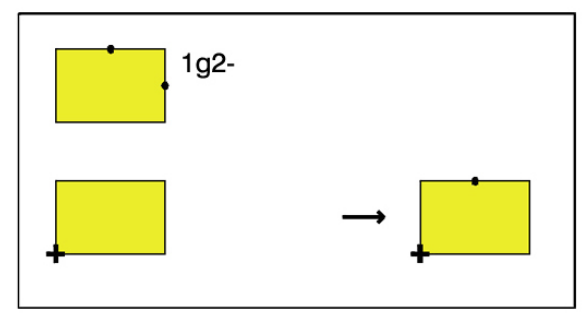

Subtractive lavout rule 4.

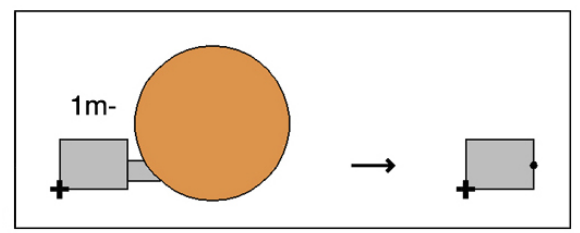

Subtractive layout rule 15.

By applying the above rules, a layout

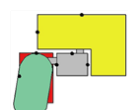

of the virtual gallery is generated for Stage 7. Relevant gallery areas and the multi-function area are removed. 
Secondly, in the application of object design rules the newly matched state labels are $\mathrm{sL}=2 \mathrm{~g} 1-, \mathrm{sL}=2 \mathrm{~g} 2-$ and $\mathrm{sL}=2 \mathrm{~m}-$. The matched object design rules are:
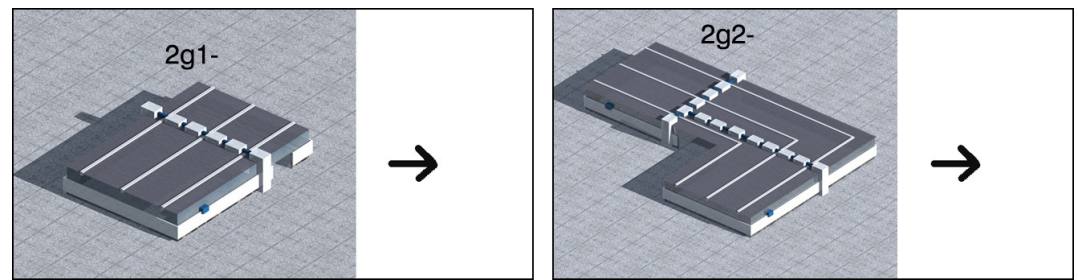

Subtractive object design rules 1 (left) and 4 (right).

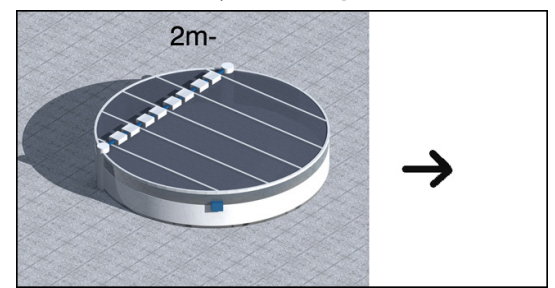

Subtractive object design rule 9 .

The above subtractive object design rules are applied to demolish the visual boundaries and visual cues of the relevant gallery areas and the multi-function area. To complete the design, the GDA searches and applies appropriate navigation rules to demolish any excessive way finding aids and hyperlinks due to the removal of the areas. No interaction rule is applied at Stage 7 because scripted behaviors are automatically removed once the objects to which they are ascribed are removed.

Figure 6.12 shows the visualization of the virtual gallery design generated for Stage 7. Compared to the design generated for Stage 6, two gallery areas (a standard gallery 1 area and an expanded gallery 2 area) and the multi-function area are removed in the current design. A reception area is also removed due to the removal of the above areas.

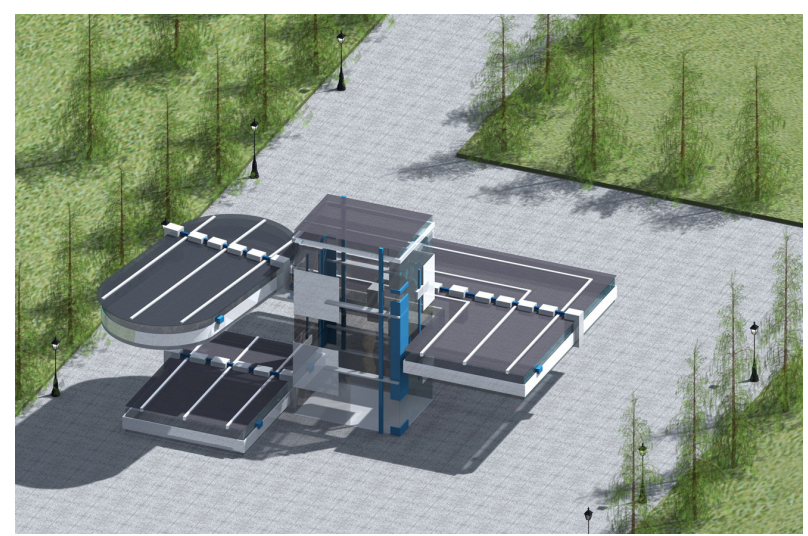

Figure 6.12: The visualization of the virtual gallery design for Stage 7. 


\subsection{Stage 8: The Artist Logs Off}

At the final stage of the design scenario the artist disconnects from the virtual world. The GDA records the current design of the virtual gallery for future references. The GDA then applies the example grammar to remove the artist' personal studio area and removes all spatial labels of the design to terminate the example grammar application, before terminating its own agent program. This generates a static design of the virtual gallery. Visitors can continue their visits to the exhibitions. The dynamic design process will re-start when the artist returns to the virtual gallery.

For the execution of the final stage, in the process of interpretation, the GDA interprets the changing needs of the artist and the visitors and changes in the virtual gallery; for example, the key change at this final stage is the artist' disconnection from the virtual world. Based on its current interpretations, in the process of hypothesizing, the GDA hypothesizes a design goal $\mathrm{O}_{\exp } \mathrm{F}=\mathrm{cS}$ (the current design of the virtual gallery is to be used as a static design and the gallery stops being dynamically designed). With the new design goal being hypothesized, new state labels can be matched for the search of eligible design rules in the example grammar for application.

Firstly, in the application of layout rules the newly matched state label is $\mathrm{SL}=1 \mathrm{cS}$. The matched layout rules are:
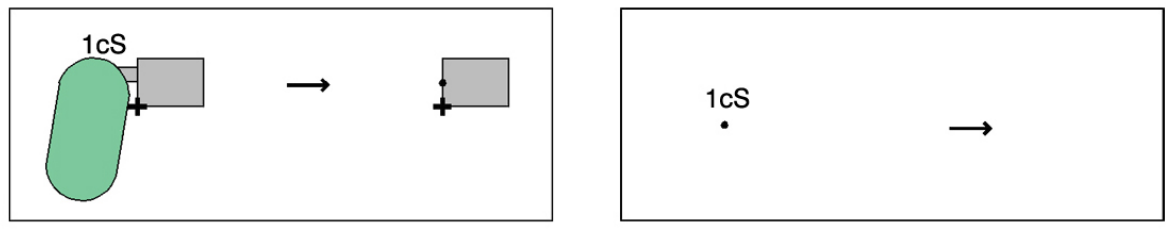

Subtractive layout rules 16 (left) and 17 (right).

The application generates the layout

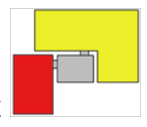
of a static design for the virtual gallery. Subtractive layout rule 16 is applied to remove the layout of the artist' personal studio area, and subtractive layout rule 17 is applied multiple times to remove all spatial labels from the design so that the application of the example grammar can be terminated.

Secondly, in the application of object design rules the newly matched state label is $\mathrm{SL}=2 \mathrm{cS}$. The matched object design rule is:

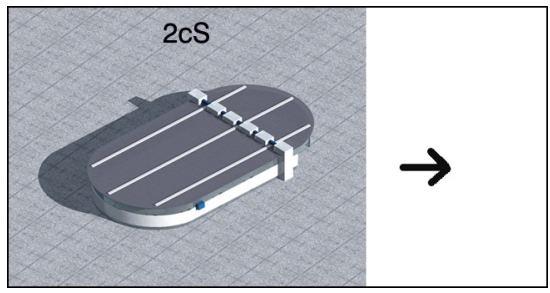

Subtractive object design rule 8. 
Subtractive object design rule 8 is applied to demolish visual boundaries and visual cues of the studio area. To complete the design, the GDA searches and applies appropriate navigation rules to remove any excessive way finding aids and hyperlinks due to the removal of the studio area. Similar to Stage 7, there is no need for interaction rules to be applied when removing scripted behaviors in the final stage.

The generated static design of the virtual gallery is visualized in Figure 6.13. The static design comprises a standard gallery 1 area, an expanded gallery 2 area, and a reception area that connects to the two gallery areas.

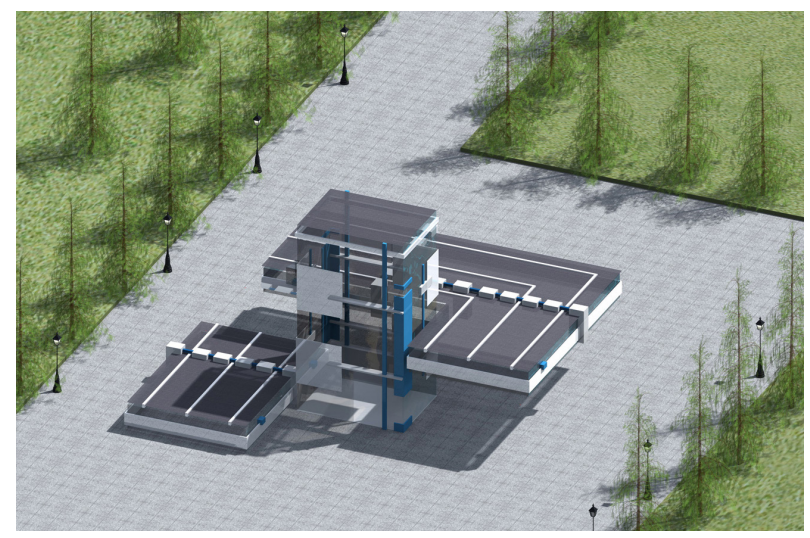

Figure 6.13: The visualization of the static virtual gallery design generated in the end of the design scenario.

\subsection{Discussion}

As demonstrated in the design scenario, the artist's GDA has dynamically generated eight designs of the virtual gallery for the eight stages of the design scenario. The virtual gallery adapts to its use through this dynamic design generation. Figure 6.14 shows how the virtual gallery changes from Stage 1 to Stage 8 . The designs of the virtual gallery generated for the scenario are visualized in sequence from the top to the bottom in the left-hand-side column of the figure. The layouts of these designs are illustrated in the right-hand-side column of the figure. As discussed above, alternative designs could have been generated if the artist had preferred to expand the virtual gallery in a different dimension at Stages 2 and 5. These alternative designs are presented in the forms of their layouts, also in the right-hand-side column. They are marked with a darker background color to contrast the ones that are generated for the scenario. 


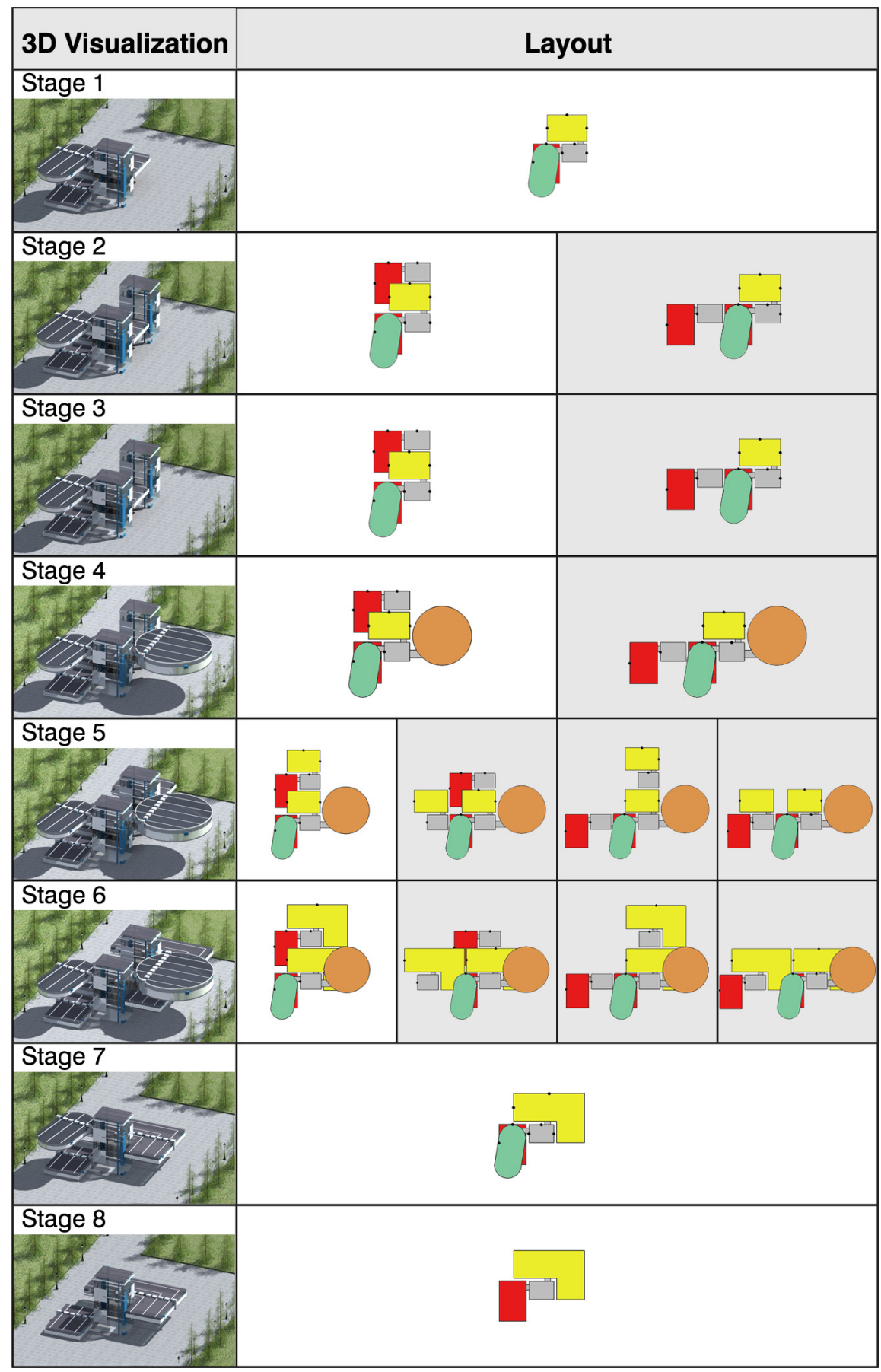

Figure 6.14: The corpus of virtual gallery designs generated for the design scenario. 


\subsubsection{Summary}

Although the design scenario is constructed in the context of a specific kind of virtual gallery for an artist and it is developed with a set of limitations as described at the beginning of the chapter, nevertheless, the scenario demonstrates the effective use of GDAs and generative design grammars both for rule-based place design in 3D virtual worlds in general.

The eight stages of the design scenario present the following changes that may occur in the virtual gallery during its use:

- Stage 1: the artist becomes present in the virtual gallery.

- Stage 2: the number of visitors increases.

- Stage 3: the artist and an invited guest plan to have a meeting.

- Stage 4: the artist plans to give an opening address.

- Stage 5: the visitors are distributed differently in the virtual gallery.

- Stage 6: the artist decides to change one of the exhibitions.

- Stage 7: the number of visitors decreases.

- Stage 8: the artist disconnects from the virtual world.

The execution of the design scenario shows that the artist's GDA reasons about the above changes and applies the example generative design grammar illustrated in Chapter 5 to dynamically design and implement the virtual gallery adapting to its use. Aiming to satisfy the GDA's current design goals, the application of the example grammar is directed by a special set of state labels so that each generated design of the virtual gallery provides appropriate areas equipped with visual boundaries, visual cues, way finding aids and hyperlinks, and other purposeful objects to support the intended activities of the virtual gallery for each stage. The comparison of the eight different virtual gallery designs generated for the eight stages of the design scenario is shown in Figure 6.14.

\subsubsection{Stylistic Characterizations of the Virtual Gallery Designs}

The design scenario demonstrates the capability of generative design grammars as a design formalism for virtual worlds. A generative design grammar is able to describe and generate a design language for virtual worlds that captures certain stylistic characterizations shared by all design instances. The corpus of virtual gallery designs generated for the design scenario presents a small set of samples from the design language defined by the example grammar. Although these virtual gallery designs are generated for different purposes, at different moments during the use of the virtual gallery, they provide a similar impression by sharing a sense of design coherency. The 
stylistic characterizations shared by these generated virtual gallery designs can be outlined from the following three aspects.

- Visualization: the virtual gallery designs generated by the example grammar are coherent in terms of the spatial relations defined for different areas of the virtual gallery, and the use of forms and color schemes for visualizing the virtual world objects.

- Navigation: the generated virtual gallery designs follow the same guidelines for providing way finding aids and hyperlinks to assist the visitors' navigation in the virtual gallery.

- Interaction: the generated virtual gallery designs also have similarities in activating object behaviors in virtual worlds. Therefore, similar experiences can be gained and applied to assist the visitors participating in activities in the virtual gallery.

\subsubsection{Technical Implementation}

In terms of the technical implementation of the design scenario, each stage of the scenario is implemented in a virtual world developed using virtual world platform Active Worlds. The GDA described in the scenario is implemented on the base of the AW agent package ${ }^{15}$. The design rules of the example grammar for supporting GDA's designing, and a general rule base for supporting the GDA's reasoning, are written using Jess ${ }^{16}$, a rule-based scripting language (Friedman-Hill, 2003). A similar implementation can also be achieved in other virtual world platforms, for example, through Linden Scripting Language (LSL) in Second Life.

\section{References}

Friedman-Hill, E. (2003), Jess in Action, Manning Publications, Greenwich, CT.

15 The AW agent package was developed by Greg Smith and Mary Lou Maher at the University of Sydney through Active Worlds Software Development Kit (SDK).

16 http://herzberg.ca.sandia.gov/jess 